\title{
Time-Shift Invariance Determines the Functional Shape of the Current in Dissipative Rocking Ratchets
}

\author{
José A. Cuesta, ${ }^{1,2, *}$ Niurka R. Quintero, ${ }^{3,4, \dagger}$ and Renato Alvarez-Nodarse ${ }^{3,5, *}$ \\ ${ }^{1}$ Grupo Interdisciplinar de Sistemas Complejos (GISC), Departamento de Matemáticas, Universidad Carlos III de Madrid, \\ Avenida de la Universidad 30, 28911 Leganés, Spain \\ ${ }^{2}$ Instituto de Biocomputación y Física de Sistemas Complejos (BIFI), Universidad de Zaragoza, 50009 Zaragoza, Spain \\ ${ }^{3}$ Instituto de Matemáticas de la Universidad de Sevilla (IMUS), Universidad de Sevilla, 41012 Sevilla, Spain \\ ${ }^{4}$ Departamento de Física Aplicada I, E.P.S., Universidad de Sevilla, Virgen de África 7, 41011, Sevilla, Spain \\ ${ }^{5}$ Departamento de Análisis Matemático, Universidad de Sevilla, Apartado 1160, 41080, Sevilla, Spain \\ (Received 25 May 2013; revised manuscript received 1 August 2013; published 18 November 2013)
}

Ratchets are devices that are able to rectify an otherwise oscillatory behavior by exploiting an asymmetry of the system. In rocking ratchets, the asymmetry is induced through a proper choice of external forces and modulations of nonlinear symmetric potentials. The ratchet currents thus obtained in systems as different as semiconductors, Josephson junctions, optical lattices, or ferrofluids show a set of universal features. A satisfactory explanation for them has challenged theorists for decades, and so far, we still lack a general theory of this phenomenon. Here, we provide such a theory by exploring-through functional analysis - the constraints that the simple assumption of time-shift invariance of the ratchet current imposes on its dependence on the external drivings. Because the derivation is based on so general a principle, the resulting expression is valid irrespective of the details and the nature of the physical systems to which it is applied, and of whether they are classical, quantum, or stochastic. The theory also explains deviations observed from universality under special conditions and allows us to make predictions of phenomena not yet observed in any experiment or simulation.

DOI: 10.1103/PhysRevX.3.041014

\section{INTRODUCTION}

Forcing nonlinear transport systems with zero-average, time-periodic, external forces may generate a ratchet current [1]. Ratchets are devices that exploit an asymmetry of the system (usually spatial) to rectify an otherwise oscillatory behavior [2-8]. The so-called rocking ratchets $[9,10]$ are able to do so by breaking a temporal symmetry-the external force cannot be reversed by a time shift-either in spatially symmetric systems [11] or in the presence of some spatial asymmetry (see, e.g., Refs. [9,12]). Ratchet currents can also be generated by a combined temporal and spatial symmetry breaking $[13,14]$.

The two most studied mechanisms to induce a net current in a rocking ratchet are harmonic mixing $[9,10]$ and gating [15-17]. In both of them, the involved periodic spatial potentials are symmetric. Harmonic mixing amounts to imposing biharmonic external forces-typically with a frequency ratio 2:1-and has been experimentally observed [18-24] and theoretically studied [25-28] in many different physical systems, both classical and

\footnotetext{
*cuesta@math.uc3m.es

niurka@us.es

†ran@us.es
}

Published by the American Physical Society under the terms of the Creative Commons Attribution 3.0 License. Further distribution of this work must maintain attribution to the author(s) and the published article's title, journal citation, and DOI.
Subject Areas: Interdisciplinary Physics, Nonlinear Dynamics

quantum. Biharmonic forces have also been used in experiments to modulate the potential in some thermal ratchet devices [29,30]. In addition, harmonic mixing with more than two harmonics has been explored in experiments with optical lattices [31,32].

Gating ratchets also need at least two harmonics to break the temporal symmetry, but they play a different role $[16,17,33]$. In the most studied setup, one of the two harmonics acts as an external force, whereas the other one is used to modulate the spatial potential $[16,17]$.

Currents generated through many different rocking ratchets share a few properties that hold regardless of the system. When two harmonics are used and their amplitudes are small, the current exhibits a shifted sinusoidal shape as a function of a precise combination of the phases of both harmonics. This sinusoid has been experimentally observed in semiconductors [18], optical lattices [20], ferrofluids [29], and Josephson junctions [21,23] and has been theoretically confirmed in studies of transport in semiconductors [18], Brownian particles [25,34], solitons [16,28,35], ferrofluids [29], and magnetic particles via dipolar interactions [30], among other systems. The phase lag of the sinusoid is known to depend on the frequency of the harmonics, the damping, and other specific parameters of the system [36,37]-accordingly, current reversals can be induced by acting on these parameters. Moreover, the ratchet current is always found to be proportional to a product of specific powers of the amplitudes of the harmonics. 
Upon increasing the amplitudes of the harmonics beyond the small limit regime, departures from the sinusoidal behavior are observed, both in experiments [33] and simulations [24]. As a consequence, current reversals can also be induced by tuning the amplitudes of the harmonics $[9,26]$.

Although there have been many theoretical attempts to explain these universal features of rocking ratchets, their scope is very limited, constrained to specific models, and only applied to harmonic mixing. For instance, stochastic theories have been used to explain the Brownian motion of a charged particle in a periodic, symmetric potential driven with a biharmonic force $[34,36]$. Also, collective coordinate theories have successfully explained harmonic mixing [28,35] and gating [16] in soliton ratchets. For several models described by nonlinear differential equations, symmetry properties of the current and of the systems can only provide conditions on the two harmonics for a ratchet current to exist $[9,11,16,27]$.

For decades, all attempts to reproduce the sinusoidal shape of the current have failed to predict the existence of a system-dependent phase lag. This lack of success is due to a flawed assumption-widely employed in the literature under the name of moment method-upon which all these theories rely. According to this method, the ratchet current can be obtained as an expansion in odd moments of the external force (starting at the third moment because the time average of the force is zero by construction). That this method is generally incorrect has been shown in Ref. [38] — where the very restricted conditions for its validity were properly delimited-but it is easy to see why in an example: If the force is a square wave, all its powers are proportional to the force itself, and therefore the current must be zero. That square-wave forces do generate nonzero currents has been shown in experiments [39], simulations [11,40], and also theoretically in Ref. [41]. The application of the moment method apparently captures the right dependence on the amplitudes in the case of harmonic mixing, but this fact is purely accidental. (For an in-depth analysis of this method and its many flaws, see Refs. $[38,41]$ and references therein.)

An alternative theoretical approach has been recently proposed for the case of harmonic mixing [38]. This theory does capture the nonzero phase lag that the ratchet current normally exhibits and also predicts a nonzero current for square-wave forces [41]. Nevertheless, despite this relative success, a general theory that encompasses a unified explanation of all universal features observed in so wide a diversity of systems, an explanation of the deviations from them that occur outside the small-amplitude regime and the effects induced by further harmonics, is still lacking. Such a theory cannot be based on the particulars of specific systems but has to rely on very general principles that hold for all of them.

In this paper, we explore the constraints that the simple time-shift invariance satisfied by the ratchet current imposes on its shape and derive an expression that explains all observations described above, both for harmonic mixing and gating ratchets (with any number of harmonics). The formula describes correctly not only the smallamplitude regime but also the deviations found for larger amplitudes. And, because it is based on so general a principle, it is valid regardless of the (dissipative) system and applicable even in the absence of a mathematical model describing the phenomenon [33]. On top of that, it allows us to make predictions so far not observed in any experiment or simulation.

Before we enter into the details, a remark seems appropriate about what this theory is not. This theory is not meant to predict when a system does exhibit a ratchet phenomenon. It would be impossible because the theory is so general that it holds both for dissipative systems that do and that do not have ratchet currents. What the theory provides is a pattern to which any ratchet current must conform. The theory claims that, under certain regularity conditions, the ratchet current-if any-must necessarily be of a given specific form. However, the pattern depends on a set of unknown, system-specific coefficients that might all be zero-hence yielding a zero current. For the same reason, the theory cannot predict any effect that depends on specific details of the system. Having made this caveat, what the theory does predict is that the current must necessarily be zero if the system possesses some specific symmetries - so it is consistent with the wellknown fact that, unless some symmetries are broken, a ratchet current cannot be generated $[9,11,27]$.

\section{GENERAL THEORY}

Suppose we have a physical system describing the position of a particle or localized structure $x(t)$ as a function of time. The system is driven by some periodic, timedependent, external driving $f(t)$ (external force, parameter modulation, etc.). Function $x(t)$-or its expectation if the system is stochastic - is uniquely determined for any given $f(t)$, and so is the ratchet current defined as

$$
v=\lim _{t \rightarrow \infty} \frac{1}{t} \int_{0}^{t} \dot{x}(\tau) d \tau=\lim _{t \rightarrow \infty} \frac{x(t)-x(0)}{t} .
$$

Mathematically, this definition means that the current $v$ is a functional of the external driving $f(t)$. Except for very specific systems in which $v$ also depends on the initial conditions (e.g., Hamiltonian systems or other nondissipative systems [14]), $v$ will—by construction-be invariant under time shifts. We will show that the fact that $v$ is a time-invariant functional of $f(t)$ is enough to determine the shape of the ratchet current for specific drivings regardless of the system under study, as long as some regularity assumptions of this functional dependence hold. Moreover, new symmetries of the system can be incorporated into the theory to further specify this shape. 


\section{A. Time-shift-invariant functionals of periodic functions}

Let $\mathcal{C}_{T}^{s}$, with $T>0$, be the set of continuous, $T$-periodic functions $\mathbf{f}: \mathbb{R} \rightarrow \mathbb{R}^{s}$, and let $\Gamma: \mathcal{C}_{T}^{s} \rightarrow \mathbb{R}$ be a real functional on $\mathcal{C}_{T}^{s}$. If $\Gamma$ is $n$ times Fréchet differentiable on $\mathcal{C}_{T}^{s}$, then it has an $n$ th-order Taylor expansion around $\mathbf{0}$ [42]. Such a Taylor expansion can be obtained as the $n$ th-order truncation of the series [43]

$$
\begin{aligned}
\Gamma[\mathbf{f}]= & \sum_{n_{1}=0}^{\infty} \cdots \sum_{n_{s}=0}^{\infty}\left\langle c_{\mathbf{n}}\left(t_{11}, \ldots, t_{1 n_{1}}, \ldots, t_{s 1}, \ldots, t_{s n_{s}}\right)\right. \\
& \left.\times f_{1}\left(t_{11}\right) \cdots f_{1}\left(t_{1 n_{1}}\right) \cdots f_{s}\left(t_{s 1}\right) \cdots f_{s}\left(t_{s n_{s}}\right)\right\rangle,
\end{aligned}
$$

where $\mathbf{n}=\left(n_{1}, \ldots, n_{s}\right)$, and we have introduced the notation

$$
\left\langle\Omega\left(t_{1}, \ldots, t_{r}\right)\right\rangle=\frac{1}{T^{r}} \int_{0}^{T} d t_{1} \cdots \int_{0}^{T} d t_{r} \Omega\left(t_{1}, \ldots, t_{r}\right) .
$$

The kernels $c_{n_{1}, \ldots, n_{s}}\left(t_{11}, \ldots, t_{s n_{s}}\right)$ are all real, $T$ periodic, and symmetric in all their arguments.

In order to avoid cumbersome expressions, we will henceforth work with the full series (2). It goes without saying that if $\Gamma$ is at most $n$ times Fréchet differentiable, the results we will obtain still hold if the series are truncated at $n$th order and an appropriate error term is added [42].

Consider the time-shift operator $\left(\mathcal{T}_{\tau} f\right)(t)=f(t+\tau)$. We will say that $\Gamma$ is invariant under time shift if $\Gamma\left[\mathcal{T}_{\tau} \mathbf{f}\right]=$ $\Gamma[\mathbf{f}]$ for all $0<\tau<T$. Time-shift invariance reflects on the kernels in Eq. (2) as the property

$c_{n_{1}, \ldots, n_{s}}\left(t_{11}-\tau, \ldots, t_{s n_{s}}-\tau\right)=c_{n_{1}, \ldots, n_{s}}\left(t_{11}, \ldots, t_{s n_{s}}\right)$

for all $0<\tau<T$.

Theorem 1.-Let $\Gamma$ be a time-shift-invariant functional with Taylor series (2), and take

$$
\mathbf{f}(t)=\left[\epsilon_{1} \cos \left(q_{1} \omega t+\phi_{1}\right), \ldots, \epsilon_{s} \cos \left(q_{s} \omega t+\phi_{s}\right)\right],
$$

where $\mathbf{q} \equiv\left(q_{1}, \ldots, q_{s}\right) \in \mathbb{N}^{s}$ is such that $\operatorname{gcd}\left(q_{1}, \ldots, q_{s}\right)=1$ [44] and $\omega=2 \pi / T$. Let $\mathcal{D}_{+}$denote the set of nonzero solutions of the Diophantine equation [45] $\mathbf{q} \cdot \mathbf{x}=$ $q_{1} x_{1}+\cdots+q_{s} x_{s}=0$, whose leftmost nonzero component is positive. Then,

$$
\Gamma[\mathbf{f}]=C_{\mathbf{0}}(\boldsymbol{\epsilon})+\sum_{\mathbf{x} \in \mathcal{D}_{+}} \epsilon_{1}^{\left|x_{1}\right|} \cdots \epsilon_{s}^{\left|x_{s}\right|} C_{\mathbf{x}}(\boldsymbol{\epsilon}) \cos \left[\mathbf{x} \cdot \boldsymbol{\phi}+\theta_{\mathbf{x}}(\boldsymbol{\epsilon})\right],
$$

where $\boldsymbol{\phi} \equiv\left(\phi_{1}, \ldots, \phi_{s}\right), \boldsymbol{\epsilon}=\left(\epsilon_{1}, \ldots, \boldsymbol{\epsilon}_{s}\right)$, and functions $C_{\mathbf{x}}(\boldsymbol{\epsilon})$ and $\theta_{\mathbf{x}}(\boldsymbol{\epsilon})$ do not depend on $\boldsymbol{\phi}$ and are even in each $\epsilon_{i}, i=1, \ldots, s$, for every $\mathbf{x} \in \mathcal{D}_{+}$.

(The proof of this theorem is deferred to Appendix A.)

When the functional $\Gamma$ exhibits further symmetries, some of the unknown functions $C_{\mathbf{x}}(\boldsymbol{\epsilon})$ and $\theta_{\mathbf{x}}(\boldsymbol{\epsilon})$ in the expansion (6) can be determined. Two symmetries are important in this respect: force reversal and time reversal.
Definition 1 (Force reversal). - Let $I \subset\{1, \ldots, s\}$ be a nonempty subset of indexes, and let $\mathbf{f}: \mathbb{R} \rightarrow \mathbb{R}^{s}$. We define the force-reversal operation $\mathcal{S}_{I}$ on $\mathbf{f}$ as the new vector function $\mathcal{S}_{I} \mathbf{f}(t)$ such that $\left(\mathcal{S}_{I} \mathbf{f}\right)_{i}(t)=-f_{i}(t)$ if $i \in I$ and $\left(\mathcal{S}_{I} \mathbf{f}\right)_{i}(t)=f_{i}(t)$ if $i \notin I$.

Corollary 1.- Under the conditions of Theorem 1, let $I \subset\{1, \ldots, s\}(I \neq \varnothing)$. Then, $\Gamma\left[\mathcal{S}_{I} \mathbf{f}\right]=-\Gamma[\mathbf{f}]$ if and only if $C_{\mathbf{x}}(\boldsymbol{\epsilon})=0$ for all $\mathbf{x} \in\{\boldsymbol{0}\} \cup \mathcal{D}_{+}$such that $\sum_{i \in I} x_{i}$ is even.

Since $C_{\mathbf{x}}(\boldsymbol{\epsilon})$ is even in all its arguments, this corollary simply follows by replacing in Eq. (6) $\epsilon_{i}$ by $-\epsilon_{i}$ for all $i \in I$.

Definition 2 (Time reversal). - Let $\mathbf{f}: \mathbb{R} \rightarrow \mathbb{R}^{s}$. We define the time-reversal operation $\mathcal{R}$ on $\mathbf{f}(t)$ as $\mathcal{R} \mathbf{f}(t)=$ $\mathbf{f}(-t)$.

Corollary 2.- Under the conditions of Theorem 1,

(a) $\Gamma[\mathcal{R} \mathbf{f}]=-\Gamma[\mathbf{f}]$ if and only if $\theta_{\mathbf{x}}(\boldsymbol{\epsilon})= \pm \pi / 2$ for each $\mathbf{x} \in \mathcal{D}_{+}$and

(b) $\Gamma[\mathcal{R} \mathbf{f}]=\Gamma[\mathbf{f}]$ if and only if $\theta_{\mathbf{x}}(\boldsymbol{\epsilon})=0$ or $\pi$ for each $\mathbf{x} \in \mathcal{D}_{+}$

The proof of this corollary follows upon realizing that time reversal amounts to replacing $\phi_{i}$ by $-\phi_{i}$, for all $i=1,2, \ldots, s$ in Eq. (6).

\section{APPLICATION TO DIFFERENT SYSTEMS}

Equation (6) has been derived under the assumption that $\Gamma$ is a sufficiently regular functional of $\mathbf{f}(t)$ and that it is time-shift invariant. Because these two assumptions are so general, it turns out that the functional form (6) must hold regardless of the specific system to which it is applied. In particular, details such as the kind of nonlinearities, whether we deal with a particle or a localized field, the actual parameters, etc., can only modify the functions $C_{\mathbf{x}}(\boldsymbol{\epsilon})$ and $\theta_{\mathbf{x}}(\boldsymbol{\epsilon})$, and only in a very specific way-they must be even functions of the amplitudes $\epsilon_{j}$. Furthermore, had the system one of the symmetries of Corollaries 1 and 2 , some of these functions would get automatically fixed regardless of any other particular. These considerations render Eq. (6) a universal expansion for the current $v$ of rocking ratchets. In what follows, we discuss its application to explain different experimental and numerical results reported in the literature of rocking ratchets.

\section{A. Two harmonic forces}

We start by considering systems for which the ratchet current arises from the combined effect of two harmonics $f_{1}(t)=\epsilon_{1} \cos \left(q \omega t+\phi_{1}\right)$ and $f_{2}(t)=\epsilon_{2} \cos \left(p \omega t+\phi_{2}\right)$. This special case is of great importance because most rocking ratchets are induced by a biharmonic force $f(t)=$ $f_{1}(t)+f_{2}(t)$ [20-29]. However, it also comprises the so-called gating ratchets $[10,16,17]$, for which $f_{1}(t)$ is an external force whereas $f_{2}(t)$ modulates the amplitude of a nonlinear potential.

For two harmonics, the Diophantine equation $\mathbf{q} \cdot \mathbf{x}=0$ becomes $q x_{1}+p x_{2}=0$. Its solutions are given by 
$\mathbf{x}=(k p,-k q), k \in \mathbb{Z}$, but those contributing to Eq. (6) have $k \in \mathbb{N}_{0}(\equiv \mathbb{N} \cup\{0\})$. Therefore, $\mathbf{x} \cdot \boldsymbol{\phi}=k\left(p \phi_{1}-\right.$ $\left.q \phi_{2}\right) \equiv k \vartheta$, and Eq. (6) reads

$v\left[f_{1}, f_{2}\right]=\sum_{k=0}^{\infty}\left(\epsilon_{1}^{p} \epsilon_{2}^{q}\right)^{k} C_{k}\left(\epsilon_{1}, \epsilon_{2}\right) \cos \left[k \vartheta+\theta_{k}\left(\epsilon_{1}, \epsilon_{2}\right)\right]$,

with $\theta_{0}\left(\epsilon_{1}, \epsilon_{2}\right)=0$.

Although Eq. (7) is valid for both ratchets induced by a biharmonic force and gating ratchets, their differences arise from their different force-reversal symmetries. Let us analyze both cases separately.

\section{B. Ratchets induced by a biharmonic force}

In rocking ratchets with symmetric spatial potentials, the current gets reversed upon reversing the force (see, e.g., Refs. [9,10,38] and references therein). Formally, $v\left[-f_{1},-f_{2}\right]=-v\left[f_{1}, f_{2}\right]$. Since $\operatorname{gcd}(p, q)=1$, either $p$ and $q$ are both odd or have a different parity. In the former case, $\left|x_{1}\right|+\left|x_{2}\right|=k(p+q)$ is always even, so, according to Corollary $1, C_{k}\left(\epsilon_{1}, \epsilon_{2}\right)=0$ for all $k \in \mathbb{N}_{0}$, and therefore $v\left[f_{1}, f_{2}\right]=0$ (i.e., there is no ratchet current). Notice that in this case, $f(t+T / 2)=-f(t)$, and since $v$ is time-shift invariant but changes sign under force reversal, it can only be 0 , hence our finding.

On the contrary, if $p+q$ is odd [in which case $f(t+\tau) \neq-f(t)$ for all $\tau>0$ ], then Corollary 1 implies only $C_{2 k}\left(\epsilon_{1}, \epsilon_{2}\right)=0, k \in \mathbb{N}$; hence,

$$
v=\sum_{\substack{k=1 \\ k \text { odd }}}^{\infty}\left(\epsilon_{1}^{p} \epsilon_{2}^{q}\right)^{k} C_{k}\left(\epsilon_{1}, \epsilon_{2}\right) \cos \left[k \vartheta+\theta_{k}\left(\epsilon_{1}, \epsilon_{2}\right)\right] .
$$

The lowest order in Eq. (8) yields

$$
v=C_{1}(0,0) \epsilon_{1}^{p} \epsilon_{2}^{q} \cos \left[\vartheta+\theta_{1}(0,0)\right]+o\left(\epsilon_{1}^{p} \epsilon_{2}^{q}\right),
$$

a result first obtained in Ref. [38].

However, Eq. (8) contains more information. The lowest order at which the next harmonic enters in $v$ is $O\left(\epsilon_{1}^{p} \epsilon_{2}^{q}\right)^{3}$. For the simplest - and most common - case studied in the literature, namely, $p=2$ and $q=1$, this fact implies that the second harmonic first appears at ninth order. Therefore, an improvement on Eq. (9) is

$v=C_{1}\left(\epsilon_{1}, \epsilon_{2}\right) \epsilon_{1}^{2} \epsilon_{2} \cos \left[\vartheta+\theta_{1}\left(\epsilon_{1}, \epsilon_{2}\right)\right]+E_{9}\left(\epsilon_{1}, \epsilon_{2}\right)$,

where the error $E_{9}\left(\epsilon_{1}, \epsilon_{2}\right)$ contains terms of order 9 or higher, and $C_{1}\left(\epsilon_{1}, \epsilon_{2}\right)$ and $\theta_{1}\left(\epsilon_{1}, \epsilon_{2}\right)$ are quadratic polynomials in $\epsilon_{1}^{2}$ and $\epsilon_{2}^{2}$. Equation (10) tells us that, whereas Eq. (9) captures the shape of the ratchet current for sufficiently small amplitudes, upon increasing the amplitudes, we can modify the phase lag $\theta_{1}\left(\epsilon_{1}, \epsilon_{2}\right)$. Put in a different way, if we fix the phases $\phi_{1}$ and $\phi_{2}$ of the biharmonic force so that $\vartheta=-\theta_{1}(0,0)+\pi / 2$, the ratchet current is
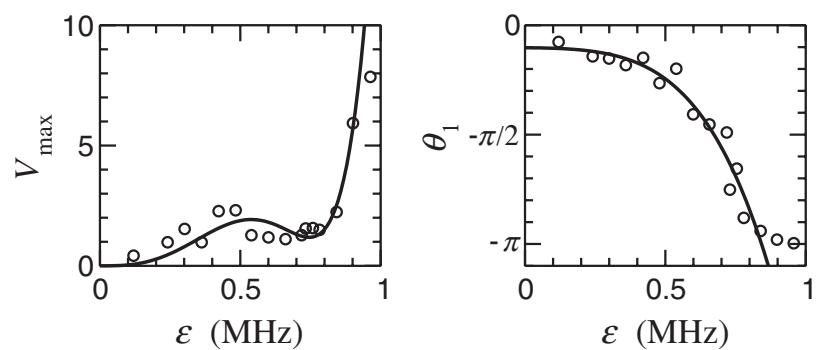

FIG. 1. Maximum ratchet velocity $v_{\max }$ and phase lag $\theta_{1}$ as functions of the amplitude $\epsilon=\epsilon_{1}=\epsilon_{2}$ of the biharmonic force, for the rocking ratchet of Ref. [24]. Points are the experimental data, and lines are fits to formula (10): $\theta_{1}(\epsilon)=-0.3238-$ $0.5996 \epsilon^{2}-4.7445 \epsilon^{4}$ and $v_{\max }(\epsilon)=\epsilon^{3}\left(39.631-124.661 \epsilon^{2}+\right.$ $\left.105.258 \epsilon^{4}\right)$.

suppressed [22,37]. But then, we can restore it without changing the phases by increasing the amplitudes.

This current reversal was observed in experiments $[22,24]$ and attributed to a dissipation-induced symmetry breaking. Our Eq. (10) reveals that this behavior is the default for a ratchet like this one because the current vanishes at a value of $\vartheta$ that depends not only on the amplitudes of the biharmonic force but also on the frequency and other parameters of the system.

Functions $C_{1}\left(\epsilon_{1}, \epsilon_{2}\right)$ and $\theta_{1}\left(\epsilon_{1}, \epsilon_{2}\right)$ are experimentally obtained for a range of values of $\epsilon_{1}=\epsilon_{2}=\epsilon$ [24]. Figure 1 shows a fit of the experimental data to the curve $v=C_{1}(\epsilon) \epsilon^{3} \cos \left[\vartheta+\theta_{1}(\epsilon)\right]$, with $v_{\max }=C_{1}(\epsilon) \epsilon^{3}$ being $C_{1}(\epsilon)$ and $\theta_{1}(\epsilon)$ quadratic polynomials in $\epsilon^{2}$.

Another prediction of the theory follows from Corollary 2: For systems having either of those two symmetries upon time reversal, all phase lags $\theta_{k}(\boldsymbol{\epsilon})$ in the expansion (8) are constant-either 0 or $\pi$, or $\pm \pi / 2$, depending on the symmetry. This fact is confirmed, e.g., by simulations carried out on the Langevin equation

$$
\alpha \dot{x}=U_{0} k \sin (2 k x)+f(t)+\eta(t)
$$

with $\eta(t)$ a zero-mean white noise such that $\left\langle\eta(t) \eta\left(t^{\prime}\right)\right\rangle=$ $D \delta\left(t-t^{\prime}\right)$ and $f(t)$ is a biharmonic force [24]. Figure 4 (upper panel) of Ref. [24] shows that $v( \pm \pi / 2)=0$ for all amplitudes. (In this overdamped regime, the velocity does not change sign upon time reversal.) This figure is especially revealing because for the largest amplitudes, the velocity clearly shows the influence of the second harmonic, and yet the phase lags remain constant.

\section{Gating ratchets}

Force reversal acts differently for gating ratchets because, of the two harmonics, only $f_{1}(t)$ is an external force. In this case, when the potential is symmetric $[10,16,17]$, we have $v\left[-f_{1}, f_{2}\right]=-v\left[f_{1}, f_{2}\right]$. Thus, Corollary 1 implies $C_{k}\left(\epsilon_{1}, \boldsymbol{\epsilon}_{2}\right)=0$ if $k p$ is even $\left(k \in \mathbb{N}_{0}\right)$. If $p$ is even, then $v=0$, whereas if $p$ is odd, then only $C_{2 k}\left(\epsilon_{1}, \epsilon_{2}\right)=0, k \in \mathbb{N}$, and we again recover 
Eqs. (8) and (9). Notice that if $p$ is even, then $q$ must be odd [because $\operatorname{gcd}(p, q)=1$ ], and therefore $f_{1}(t+T / 2)=$ $-f_{1}(t)$ and $f_{2}(t+T / 2)=f_{2}(t)$. Thus, a time shift can reverse the current-which means that the current must be zero.

Thus, the ratchet currents produced by either gating or a biharmonic force are both given by the same formula. There is an exception, though: Gating does not put any constraint on $q$, so a ratchet current can be obtained even for $q=p=1[10,16,17]$. For this particular case, the lowest order at which the second harmonic shows up in the current is the sixth, i.e.,

$v=C_{1}\left(\epsilon_{1}, \epsilon_{2}\right) \epsilon_{1} \epsilon_{2} \cos \left[\vartheta+\theta_{1}\left(\epsilon_{1}, \epsilon_{2}\right)\right]+E_{6}\left(\epsilon_{1}, \epsilon_{2}\right)$,

and $C_{1}\left(\epsilon_{1}, \epsilon_{2}\right)$ and $\theta_{1}\left(\epsilon_{1}, \epsilon_{2}\right)$ are linear in $\epsilon_{1}^{2}$ and $\epsilon_{2}^{2}$. Accordingly, a shift of the phase lag with the amplitudes similar to that observed in biharmonic ratchets [24] is to be expected in gating ratchets. Thus, not only has formula (12) been obtained here for the first time (to the best of our knowledge, no theory has ever been attempted to explain the current observed in gating ratchets) but the possibility of inverting the current by varying the amplitudes of the harmonics in these systems is a prediction of this theory that, as far as we know, still needs experimental confirmation.

\section{Particles moving in asymmetric potentials}

An interesting case to analyze with the theory is that of particles moving (or solitons lying) in potentials lacking mirror symmetry. In these cases, the current does not have the force-reversal symmetry exploited above because the mirror image of the system is a different system. Then, all terms in Eq. (7) are nonzero in principle. In the case of two harmonics-irrespective of whether we are considering ratchets induced by biharmonic forces or gating ratchets - the lowest order in the expansion (7) is given by $C_{0}\left(\epsilon_{1}, \epsilon_{2}\right)$, a polynomial of $\epsilon_{1}^{2}$ and $\epsilon_{2}^{2}$. Clearly, $C_{0}(0,0)=0$ if there is no ratchet current in the absence of external force; therefore, in this case, the theory predicts, for small amplitudes, a ratchet current independent of the phases (a dependence that may be restored at higher orders) and proportional to a linear combination of $\epsilon_{1}^{2}$ and $\epsilon_{2}^{2}$.

As a matter of fact, the theory also predicts that even with a single harmonic (say, $\epsilon_{2}=0$ ), a ratchet current proportional to $\epsilon_{1}^{2}$ can be generated. This proportionality is indeed what was found in Refs. [9,46]. In this case, we also know from Eq. (7) that all higher-order terms are identically zero, so the prediction is even stronger: The current must be of the form $\epsilon_{1}^{2} Q\left(\epsilon_{1}^{2}\right)$, with $Q(x)$ a certain function. Notice, in particular, that, depending on whether $Q(x)$ does or does not change sign, the current may or may not exhibit reversals upon variations of the amplitude $\epsilon_{1}$.

\section{E. Other ratchets with two harmonics}

Liquid drops on a horizontal plate exhibit ratchet movement when the plate is vibrated with both horizontal and vertical harmonic forces [33]. These forces have the same frequency and a relative phase $\phi$, and as usual, the ratchet current depends on $\phi$. We are not aware of any theoretical approach that explains why the average velocity $v$ of the drops exhibits a nonsinusoidal behavior as a function of the relative phase shift $\phi$. However, Fig. 3(a) of Ref. [33] reveals that $v$ changes sign when the vertical force $f_{1}$ is reversed; i.e., $v\left[-f_{1}, f_{2}\right]=-v\left[f_{1}, f_{2}\right]$. According to our approach, this property is enough to conclude that the drop velocity must behave as the current of a gating ratchet. Hence, it will be given by Eq. (8) for $p=q=1$. Figure 2 shows a fit with the first two harmonics of this equation to the experimental data of Ref. [33].

This anharmonicity is also predicted by our theory when the ratchet is induced by a biharmonic force with large amplitudes, and it has been reported recently in simulations of classical particles in a one-dimensional driven superlattice [47].

\section{F. Forcing with more than two harmonics}

In some experiments with cold atoms [31,32], ratchets are generated using more than two harmonics. The simplest one is of the form

$$
\begin{aligned}
f(t)= & a\left[\cos \left(q \omega t+\phi_{1}\right)+\cos \left(2 q \omega t+\phi_{2}\right)\right. \\
& \left.+\cos \left(p \omega t+\phi_{3}\right)\right] .
\end{aligned}
$$

Although the Diophantine equation $q x_{1}+2 q x_{2}+p x_{3}=$ 0 has three unknowns, the solution can be readily obtained using Blankinship's algorithm [48] as $\mathbf{x}=k_{1} \mathbf{s}_{1}+k_{2} \mathbf{s}_{2}$, where $\mathbf{s}_{1}=(2,-1,0), \mathbf{s}_{2}=(p, 0,-q)$, and $k_{1}, k_{2} \in \mathbb{Z}$. Hence, $\mathbf{x}=\left(2 k_{1}+p k_{2},-k_{1},-q k_{2}\right)$. The subset contributing to Eq. (6) is defined by $2 k_{1}+p k_{2} \in \mathbb{N}_{0}$; on the other hand, because of force reversal (cf. Corollary 1), the only

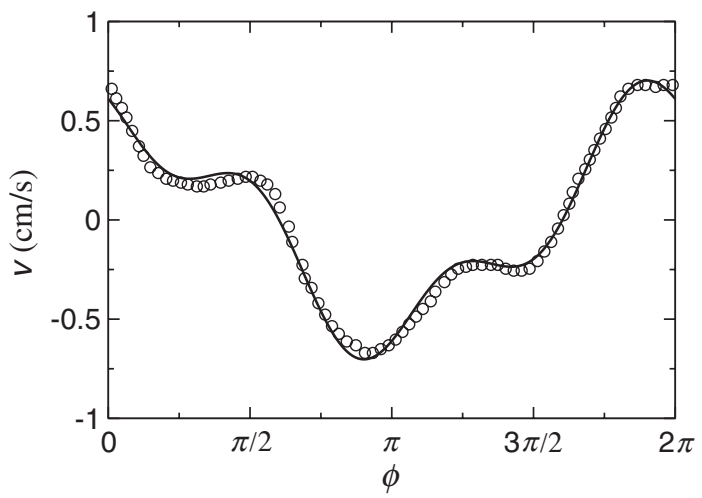

FIG. 2. Droplet velocity $v$ as function of the phase shift $\phi$ between the horizontal and vertical vibrations of the plate. Symbols represent experimental data from Fig. 3, upper panel, $A_{h}=240 \mu \mathrm{m}$, of Ref. [33]. The line represents the fit of the curve $v(\phi)=0.5435 \cos (\phi-0.0211)+0.1972 \cos (3 \phi+1.2267)$. 
nonzero coefficients have $3 k_{1}+(p+q) k_{2}$ odd. Hence, if $p+q$ is even, then $k_{1}$ must be odd, whereas if $p+q$ is odd, then $k_{1}+k_{2}$ must be odd. Then,

$$
\begin{aligned}
v= & \sum_{\mathbf{k} \in \Omega_{p, q}} C_{\mathbf{k}}(a) a^{2 k_{1}+p k_{2}+\left|k_{1}\right|+q\left|k_{2}\right|} \\
& \times \cos \left[k_{1} \boldsymbol{\vartheta}_{1}+k_{2} \vartheta_{2}+\theta_{\mathbf{k}}(a)\right],
\end{aligned}
$$

where $\vartheta_{1}=2 \phi_{1}-\phi_{2}, \vartheta_{2}=p \phi_{1}-q \phi_{3}$, and $\Omega_{p, q}=$ $\left\{\left(k_{1}, k_{2}\right) \in \mathbb{Z}^{2}: 2 k_{1}+p k_{2} \geq 0, k_{1}\right.$ odd $\}$ if $p+q$ is even, or $\Omega_{p, q}=\left\{\left(k_{1}, k_{2}\right) \in \mathbb{Z}^{2}: 2 k_{1}+p k_{2} \geq 0, k_{1}+k_{2}\right.$ odd $\}$ if $p+q$ is odd.

The choice $q=p=1$ [31] reduces Eq. (13) to a biharmonic force where the amplitude of the one of harmonics depends on the phase $\phi_{3}$. In other words, the shape of the current is again a sinusoidal function of $\vartheta_{1}$, with the usual cubic prefactor of the amplitudes; however, in this case, both the maximum current and the phase lag depend on $\phi_{3}$. This dependence is exactly what the experiments reveal (cf. Fig. 1 of Ref. [31]).

Another relevant choice of parameters is $q, p \rightarrow \infty$ and $q \neq p$ [31]. For $a<1$, it implies that $k_{2}=0$. Thus, regardless of the parity of $p+q$, Eq. (14) becomes

$$
v=\sum_{\substack{k=1 \\ k \text { odd }}}^{\infty} C_{k, 0}(a) a^{3 k} \cos \left[k \vartheta_{1}+\theta_{k, 0}(a)\right] .
$$

The lowest order is $v=C_{1,0}(0) a^{3} \cos \left[\vartheta_{1}+\theta_{1,0}(0)\right]+O\left(a^{5}\right)$, which explains the observations made in Ref. [31], namely, the sinusoidal dependence on $\vartheta_{1}$ and the insensitivity of $\theta_{1,0}(0)$ to variations of the phase $\phi_{3}$.

The limit case $p, q \rightarrow \infty$ is particularly interesting because it connects the effect of perturbations with quasiperiodic forces. Suppose the harmonics depend on two frequencies $\omega_{1}$ and $\omega_{2}$ such that $\omega_{2} / \omega_{1}$ is not a rational number. One can choose rational approximants $p / q$ of $\omega_{2} / \omega_{1}$ such that $\omega_{1} \approx q \omega$ and $\omega_{2} \approx p \omega$ for a suitable $\omega$. The theory can then be applied for each choice of $p$ and $q$, and the quasiperiodic limit can be recovered as the limit $p, q \rightarrow \infty$ and $\omega \rightarrow 0$ with $p / q \rightarrow \omega_{2} / \omega_{1}$. For an illustration of the application of this method, we refer to the appendix of Ref. [49].

A second more complicated forcing has also been tested for cold atoms [32]. The force in this case can be cast as a sum of four harmonics $f(t)=f_{1}(t)+f_{2}(t)+f_{3}(t)+$ $f_{4}(t)$, where

$$
\begin{aligned}
f_{1}(t) & =\frac{b}{2}(2 q+p) \cos \left[(2 q+p) \omega t+2 \phi_{1}+\phi_{2}\right], \\
f_{2}(t) & =\frac{b}{2}(2 q-p) \cos \left[(2 q-p) \omega t+2 \phi_{1}-\phi_{2}\right], \\
f_{3}(t) & =\frac{a}{2}(q+p) \cos \left[(q+p) \omega t+\phi_{1}+\phi_{2}\right], \\
f_{4}(t) & =\frac{a}{2}(q-p) \cos \left[(q-p) \omega t+\phi_{1}-\phi_{2}\right] .
\end{aligned}
$$

Two cases have been studied [32]: $q=p=1$ and $q=3$, $p=2$.

For $q=p=1, f_{4}(t)=0$, and there are three harmonics left. The expansion of $v$ in terms of $\vartheta=\phi_{2}-\phi_{1}$ and the amplitudes can be obtained using a similar procedure [see Appendix B, Eq. (B1)]. To lowest order,

$$
v=b^{2} a\left\{C \cos \left(3 \vartheta+\theta_{0}\right)+D \cos \left(\vartheta+\theta_{1}\right)\right\}+E_{5}(a, b),
$$

where $E_{5}(a, b)$ contains fifth-order terms in $a$ and $b$. This expression features, even at the lowest order in the amplitudes, a deviation from the usual sinusoidal shape. In the experiments, the second harmonic went unnoticed because at that time no available theory predicted any such deviation. However, the fit of the experimental data to a cosine function shows a systematic discrepancy that might be the fingerprint of this second harmonic (cf. Fig. 1 of Ref. [32]). Further experiments should reveal this second harmonic more clearly.

The second case experimentally tested is $q=3, p=2$. For this case, all four harmonics (16) are present. The full expansion in terms of $\vartheta=3 \phi_{2}-2 \phi_{1}$ and the amplitudes is obtained in Appendix B [cf. Eq. (B2)]. To lowest order,

$$
v=A(a, b) \cos [\vartheta+\psi(a, b)]+E_{5}(a, b),
$$

with $A(a, b)$ and $\psi(a, b)$ given by Eq. (B4). The usual cosine shape of the current was already observed in the experiments [32]. However, Eq. (18) reveals an unexpected new effect. In harmonic mixing currents, it is customary to set $a=r \epsilon$ and $b=(1-r) \epsilon$ and vary $0 \leq r \leq 1$. If the system is driven by a biharmonic force, changing $r$ changes the intensity of the current [20]. However, if $\epsilon$ is sufficiently small, the phase at which the current vanishes does not depend on $r$. In other words, if $\vartheta$ is fixed to this phase and $r$ is varied, no ratchet current is produced. (As explained before [cf. Eq. (10)], for $\vartheta$ to vary with $r$, the amplitude $\epsilon$ must be large; see also Ref. [24].) However, Eq. (18) tells us that $\psi(a, b)$ does depend on $r$ even for small $\epsilon$. Therefore, by setting $\vartheta$ so that the current is zero for a given $r$, we can generate a ratchet current by simply changing $r$.

To confirm this prediction of the theory, we carry out simulations for the damped sine-Gordon equation

$$
\psi_{t t}-\psi_{x x}+\sin \psi+\beta \psi_{t}=f(t)
$$

driven by the multifrequency force (16) with $q=3, p=2$. We have solved numerically this equation in the interval $[-70,70]$, with periodic boundary conditions, by discretizing the second spatial derivative using centered finite differences on a grid of step size $\Delta x=0.1$. We have integrated the resulting set of ordinary differential equations with a fourth-order Runge-Kutta method along 10 complete periods, with a time step $\Delta t=0.01$. As the initial condition, we use an exact static one-soliton solution, centered at zero, of the unforced $[f(t)=0]$ and undamped $(\beta=0)$ sine-Gordon equation (19). 


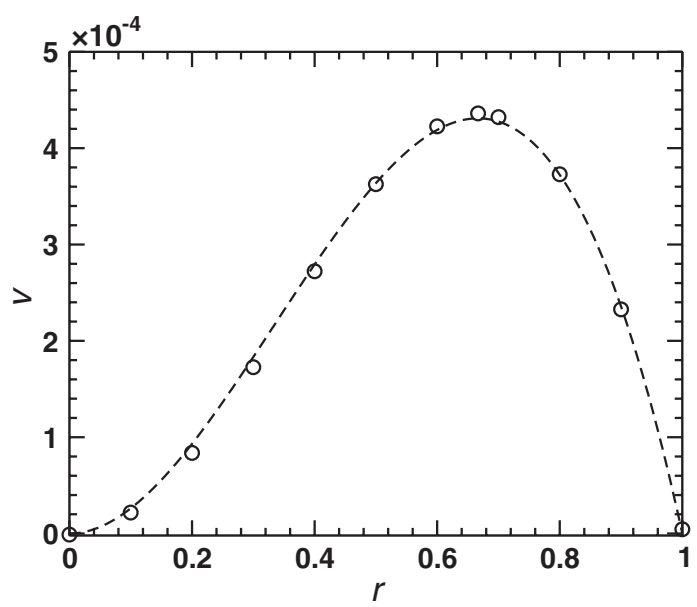

FIG. 3. Ratchet effect induced in a damped sine-Gordon system [cf. Eq. (19)] driven by the force (16) upon changing the amplitudes $a=r \epsilon$ and $b=(1-r) \epsilon$. The velocity $v$ is plotted as a function of $r$. Parameters are $\omega=0.05, \phi_{1}=\pi / 2, \beta=$ 0.05 , and $\epsilon=0.03$. Phase $\phi_{2}=0.64486$ is chosen so that $v$ vanishes for $r=0$. Circles are results obtained from the simulations. The line is the fitted curve $v=0.0029089(1-r) r^{2}$.

Notice that if $r=0$ or $r=1$, only two of the four harmonics (16) remain. Their frequencies are such that for $r=1, f(t)=-f(t+T / 2)$-hence, there is no ratchet current-whereas for $r=0$, this symmetry is brokenhence, there is a ratchet current. Accordingly, we set $r=0$ and $\phi_{1}=\pi / 2$, and find the value of $\phi_{2}$ for which $v=0$. Then, we fix this value for $\phi_{2}$ and vary $r$. The result is shown in Fig. 3. As predicted, varying $r$ induces a ratchet current. As a matter of fact, the numerical values fit perfectly the theoretical prediction $v \propto \epsilon^{3}(1-r) r^{2}$ that follows from Eqs. (18) and (B3).

\section{DISCUSSION AND CONCLUSIONS}

In this work, we have introduced a theory that captures, in a unified framework, the ratchet transport generated by zero-average, periodic drivings of very different kinds of systems, like cold atoms in optical lattices, fluxons in Josephson junctions, current in semiconductors, or transport of ferromagnetic nanoparticles in liquids. The theory can be applied to classical or quantum dissipative systems alike, with or without thermal fluctuations. The number of different harmonics the theory can deal with is arbitrary. Although most studies use two, added up in a single biharmonic force or used for two different purposes (like a force and a potential modulation [16,17] or two independent forces [33]), the theory also explains experiments carried out driving the system with three or four harmonics [31,32], as well as experiments in ferrofluids, where the biharmonic force modulates the potential in a new type of thermal ratchet device [29].

Focusing on the results for two harmonics, Eq. (7) already captures many universal features observed in experiments and simulations. First, it shows the widespread sinusoidal dependence observed when the amplitude of the external forces is small [16-18,20,21,23,26,28,29]. Second, it explains why the sinusoid is observed even when the amplitude of the force is not so small [24]. Third, it captures the departures of this sinusoidal shape for even larger amplitudes [24,33]. And fourth, it shows that the point where the current vanishes (the phase lag) depends on the amplitude, the frequency, and the rest of the system parameters. In particular, this dependence implies that we can generate or revert the current by simply changing the amplitudes of the two harmonics [24,33], their frequency $[36,50]$, or (rather paradoxically) the damping in systems with dissipation $[22,36,41,51]$. If the system satisfies certain symmetries, the theory predicts that the phase lags can no longer be modified by changing the amplitudes of the harmonics (Corollary 2), in agreement with what is observed in some equations for particles or solitons moving in a nonlinear potential and in certain experiments $[23,25,29,41]$.

One of the most remarkable facts about this theory is its universality. In its derivation, we have simply used two assumptions: (a) The velocity is a sufficiently regular functional of the external force (regularity condition) and (b) it is invariant under time shifts (time-shift symmetry). Assumption (a) is used to make a Taylor expansionperhaps only up to some finite order-of the velocity with respect to the external force; assumption (b) leads, in the case of harmonic forcings, to a Fourier expansion in terms of some combination of the phase shifts between the harmonics. The fact that the functional form (6) is obtained under so general assumptions implies that the particulars of the system under study (e.g., the kind of nonlinearities or the specific parameters) can only tune the constants but never change the functional form. As a matter of fact, we do not even need to have an explicit mathematical model of the experimental system to predict how the velocity depends on the phases of the harmonics and to constrain its dependence on the amplitudes (e.g., the case analyzed in Fig. 2).

Of the two assumptions above, only regularity limits the applicability of the theory. Besides, it might be a requirement that is hard to verify for a given physical system. Nonetheless, the success of the theory in explaining the results of so many different experimental and numerical sources suggests that the systems to which it does not apply must be rare. Exceptions can be found, though. For instance, simulations of the discrete Frenkel-Kontorova system show discontinuities in the behavior of the current as a function of the phases in the biharmonic force [52]. Also, the ratchet current of periodically forced overdamped particles moving in an asymmetric potential exhibits discontinuities as a function of the amplitude of the forcing [11,53] —although these discontinuities disappear in the presence of noise, which may thus be acting as a regularizer of the functional. 
We end by pointing out that universality permits us not only to explain a plethora of specific phenomena or anomalies that different experiments and simulations have evidenced but also to predict new ones that have not been observed yet and need experimental confirmation. Some of them are described above, and some others have been stated along the way while analyzing systems which had been experimentally studied. However, by making specific choices for the number of harmonics and their frequencies in Eq. (6), many more can be derived.

\section{ACKNOWLEDGMENTS}

We thank Franck Celestini and Ferruccio Renzoni for providing us their experimental data. We acknowledge financial support through Grants No. MTM2012-36732C03-03 (R. A.-N.), No. FIS2011-24540 (N.R.Q.), PRODIEVO, and Complexity-Net RESINEE (J. A.C.), from Ministerio de Economía y Competitividad (Spain); Grants No. FQM262 (R. A.-N.), No. FQM207 (N. R. Q.), No. FQM-7276, and No. P09-FQM-4643 (N. R. Q., R. A.-N.), from Junta de Andalucía (Spain); Project MODELICO-CM (J.A.C.), from Comunidad de Madrid (Spain); and a grant from the Humboldt Foundation through a Research Fellowship for Experienced Researchers No. SPA 1146358 STP (N. R. Q.).

\section{APPENDIX A: PROOF OF THEOREM 1}

Writing the cosines as complex exponentials and substituting in Eq. (2) leads to

$$
\Gamma[\mathbf{f}]=\sum_{\mathbf{k}, l \in \mathbb{N}_{0}^{s}} \epsilon_{1}^{k_{1}+l_{1}} \cdots \epsilon_{s}^{k_{s}+l_{s}} A(\mathbf{k}, \boldsymbol{l}) e^{i(\mathbf{k}-l) \cdot \boldsymbol{\phi}},
$$

where

$$
\begin{aligned}
A(\mathbf{k}, \boldsymbol{l})= & \prod_{j=1}^{s} \frac{\left(k_{j}+l_{j}\right) !}{2^{k_{j}+l_{j}} k_{j} ! l_{j} !} \\
& \times \hat{c}_{\mathbf{k}+\boldsymbol{l}}\left(\left\{q_{1}\right\}_{k_{1}},\left\{-q_{1}\right\}_{l_{1}}, \ldots,\left\{q_{s}\right\}_{k_{s}},\left\{-q_{s}\right\}_{l_{s}}\right) .
\end{aligned}
$$

Here, we are using the shorthand $\{a\}_{k}=\overbrace{a, \ldots, a}^{k \text { times }}$ and denoting

$\hat{c}_{\mathbf{n}}\left(r_{1}, \ldots, r_{|\mathbf{n}|}\right)=\left\langle c_{\mathbf{n}}\left(t_{1}, \ldots, t_{|\mathbf{n}|}\right) e^{i \omega\left(r_{1} t_{1}+\cdots+r_{|\mathbf{n}|} t_{|\mathbf{n}|}\right)}\right\rangle$,

with $|\mathbf{n}|=n_{1}+\cdots+n_{s}$. The combinatorial factors in Eq. (A2) arise from the symmetry in the arguments of the kernels. Notice that the definition (A3) leads to

$$
A(\mathbf{k}, l)=\overline{A(l, \mathbf{k})} .
$$

Now, making use of the time-shift invariance (4) in Eq. (A3) implies that $A(\mathbf{k}, \boldsymbol{l})=0$ whenever $\mathbf{q} \cdot(\mathbf{k}-\boldsymbol{l}) \neq 0$. Therefore, the only indexes $\mathbf{k}, \boldsymbol{l} \in \mathbb{N}_{0}^{s}$ in Eq. (A1) that can contribute to $\Gamma[\mathbf{f}]$ are those whose difference is a solution of the Diophantine equation $\mathbf{q} \cdot \mathbf{x}=0$. The set of solutions of this equation $\mathcal{D}$ can be decomposed as
$\mathcal{D}=\{\boldsymbol{0}\} \cup \mathcal{D}_{+} \cup\left(-\mathcal{D}_{+}\right)$. Now, for every $\mathbf{x} \in \mathcal{D}_{+}$, let us define $\mathbf{m}=\left(m_{1}, \ldots, m_{s}\right)$ such that

$$
m_{j}= \begin{cases}l_{j} & \text { if } x_{j} \geq 0 \\ k_{j} & \text { if } x_{j}<0 .\end{cases}
$$

Thus, if $x_{j} \geq 0$, we can set $l_{j}=m_{j}$ and $k_{j}=m_{j}+x_{j}$, whereas if $x_{j}<0$, we can set $k_{j}=m_{j}$ and $l_{j}=m_{j}-x_{j}$. Denoting $B(\mathbf{m}, \mathbf{x})=A(\mathbf{k}, \boldsymbol{l})$, Eq. (A1) becomes

$$
\begin{aligned}
\Gamma[\mathbf{f}]= & \sum_{\mathbf{m} \in \mathbb{N}_{0}^{s}}\left(\prod_{j=1}^{s} \epsilon_{j}^{2 m_{j}}\right) B(\mathbf{m}, \mathbf{0}) \\
& +\sum_{\mathbf{m} \in \mathbb{N}_{0}^{s}} \sum_{\mathbf{x} \in \mathcal{D}_{+}}\left(\prod_{j=1}^{s} \epsilon_{j}^{2 m_{j}+\left|x_{j}\right|}\right) B(\mathbf{m}, \mathbf{x}) e^{i \mathbf{x} \cdot \boldsymbol{\phi}} \\
& +\sum_{\mathbf{m} \in \mathbb{N}_{0}^{s}} \sum_{\mathbf{x} \in \mathcal{D}_{+}}\left(\prod_{j=1}^{s} \epsilon_{j}^{2 m_{j}+\left|x_{j}\right|}\right) B(\mathbf{m},-\mathbf{x}) e^{-i \mathbf{x} \cdot \boldsymbol{\phi}} .
\end{aligned}
$$

Taking into account that Eq. (A4) implies $B(\mathbf{m},-\mathbf{x})=$ $\overline{B(\mathbf{m}, \mathbf{x})}$, if we define

$$
C_{\mathbf{x}}(\boldsymbol{\epsilon}) e^{i \theta_{\mathbf{x}}(\boldsymbol{\epsilon})}=\sum_{\mathbf{m} \in \mathbb{N}_{0}^{s}}\left(\prod_{j=1}^{s} \epsilon_{j}^{2 m_{j}}\right) B(\mathbf{m}, \mathbf{x}),
$$

with $C_{\mathbf{x}}(\boldsymbol{\epsilon})=C_{-\mathbf{x}}(\boldsymbol{\epsilon}) \geq 0$ and $\theta_{\mathbf{x}}(\boldsymbol{\epsilon})=-\theta_{-\mathbf{x}}(\boldsymbol{\epsilon}) \in \mathbb{R}$, we finally obtain Eq. (6).

\section{APPENDIX B: FORCING WITH THREE OR FOUR HARMONICS}

When $q=p=1$ in Eq. (16), the frequency, amplitude, and phase vectors of the three nonzero harmonics are $\mathbf{q}=$ $(3,1,2), \boldsymbol{\epsilon}=(3 b / 2, b / 2, a)$, and $\boldsymbol{\phi}=\left(2 \phi_{1}+\phi_{2}, 2 \phi_{1}-\right.$ $\left.\phi_{2}, \phi_{1}+\phi_{2}\right)$. Blankinship's algorithm applied to $\mathbf{q} \cdot \mathbf{x}=0$ yields $\mathbf{x}=k_{1}(1,-3,0)+k_{2}(0,2,-1)=\left(k_{1}, 2 k_{2}-3 k_{1},-k_{2}\right)$. Then, $\mathbf{x} \cdot \boldsymbol{\phi}=\left(4 k_{1}-3 k_{2}\right) \vartheta$, where $\vartheta \equiv \phi_{2}-\phi_{1}$. Force reversal imposes $k_{2}-2 k_{1}$ to be odd, which means that $k_{2}$ must be odd. Thus, the expansion of the ratchet velocity will be

$$
\begin{aligned}
v= & \sum_{\substack{k_{2}=1 \\
k_{2} \text { odd }}}^{\infty} \tilde{C}_{0, k_{2}}(a, b)\left(b^{2} a\right)^{k_{2}} \cos \left[3 k_{2} \vartheta-\theta_{0, k_{2}}(a, b)\right] \\
& +\sum_{\substack{k_{1}=1 \\
k_{2} \text { odd } \\
k_{2}, \mathbb{Z}}}^{\infty} \tilde{C}_{k_{1}, k_{2}}(a, b) b^{k_{1}+\left|2 k_{2}-3 k_{1}\right|} a^{\left|k_{2}\right|} \\
& \times \cos \left[\left(4 k_{1}-3 k_{2}\right) \vartheta+\theta_{k_{1}, k_{2}}(a, b)\right],
\end{aligned}
$$

where $\tilde{C}_{k_{1}, k_{2}}(a, b) \equiv 3^{k_{1}} 2^{-k_{1}-\left|2 k_{2}-3 k_{1}\right|} C_{k_{1}, k_{2}}(a, b)$. Hence, the lowest order in this expansion is of the form (17).

For the case $q=3, p=2$, in Eq. (16), the frequency, amplitude, and phase vectors are $\mathbf{q}=(8,4,5,1), \boldsymbol{\phi}=$ $\left(2 \phi_{1}+\phi_{2}, 2 \phi_{1}-\phi_{2}, \phi_{1}+\phi_{2}, \phi_{1}-\phi_{2}\right)$, and $\boldsymbol{\epsilon}=(4 b, 2 b$, $5 a / 2, a / 2)$, and the solution of $\mathbf{q} \cdot \mathbf{x}=0$ is $\mathbf{x}=k_{1}(1,0,0,-8)+k_{2}(0,1,0,-4)+k_{3}(0,0,1,-5)=\left(k_{1}, k_{2}\right.$, $\left.k_{3},-8 k_{1}-4 k_{2}-5 k_{3}\right)$. Thus, $\mathbf{x} \cdot \boldsymbol{\phi}=\left(3 k_{1}+k_{2}+2 k_{3}\right) \vartheta$, 
where $\vartheta=3 \phi_{2}-2 \phi_{1}$. Force reversal requires $7 k_{1}+$ $3 k_{2}+4 k_{3}=k_{1}+k_{2}+2\left(3 k_{1}+k_{2}+2 k_{3}\right)$ to be odd; in other words, $k_{1}+k_{2}$ must be odd. Hence,

$$
\begin{aligned}
v= & \sum_{\substack{k_{2}=\\
k_{2} \text { odd }}}^{\infty} \sum_{k_{3} \in \mathbb{Z}} \tilde{C}_{0, k_{2}, k_{3}}(a, b) a^{\left|k_{3}\right|+\left|4 k_{2}+5 k_{3}\right|} b^{k_{2}} \\
& \times \cos \left[\left(k_{2}+2 k_{3}\right) \vartheta+\theta_{0, k_{2}, k_{3}}(a, b)\right] \\
& +\sum_{k_{1}=1}^{\infty} \sum_{\substack{k_{2} \in \mathbb{Z} \\
k_{1}+k_{2} \text { odd }}}^{\infty} \sum_{k_{3} \in \mathbb{Z}} \tilde{C}_{k_{1}, k_{2}, k_{3}}(a, b) a^{\left|k_{3}\right|+\left|8 k_{1}+4 k_{2}+5 k_{3}\right|} b^{k_{1}+\left|k_{2}\right|} \\
& \times \cos \left[\left(3 k_{1}+k_{2}+2 k_{3}\right) \vartheta+\theta_{k_{1}, k_{2}, k_{3}}(a, b)\right],
\end{aligned}
$$

where $\quad \tilde{C}_{\mathbf{k}}(a, b) \equiv 5^{\left|k_{3}\right|} 2^{2 k_{1}+\left|k_{2}\right|-\left|k_{3}\right|-\left|8 k_{1}+4 k_{2}+5 k_{3}\right|} C_{\mathbf{k}}(a, b)$. The lowest order of this expansion is

$$
\begin{aligned}
v= & \tilde{C}_{0,1,-1}(0,0) b a^{2} \cos \left[\vartheta-\theta_{0,1,-1}(0,0)\right] \\
& +\tilde{C}_{1,-2,0}(0,0) b^{3} \cos \left[\vartheta+\theta_{1,-2,0}(0,0)\right] \\
& +E_{5}(a, b),
\end{aligned}
$$

where $E_{5}(a, b)$ contains terms of fifth order in $a$ and $b$. This expression can be rewritten as in Eq. (18) by defining

$$
\begin{aligned}
A(a, b) e^{i \psi(a, b)} \equiv & \tilde{C}_{0,1,-1}(0,0) b a^{2} e^{-i \theta_{0,1,-1}(0,0)} \\
& +\tilde{C}_{1,-2,0}(0,0) b^{3} e^{i \theta_{1,-2,0}(0,0)}
\end{aligned}
$$

[1] D. Cole, S. Bending, S. Savel'ev, A. Grigorenko, T. Tamegai, and F. Nori, Ratchet without Spatial Asymmetry for Controlling the Motion of Magnetic Flux Quanta Using Time-Asymmetric Drives, Nat. Mater. 5, 305 (2006).

[2] I. Zapata, R. Bartussek, F. Sols, and P. Hänggi, Voltage Rectification by a SQUID Ratchet, Phys. Rev. Lett. 77, 2292 (1996).

[3] F. Falo, P. J. Martínez, J. J. Mazo, and S. Cilla, Ratchet Potential for Fluxons in Josephson-Junction Arrays, Europhys. Lett. 45, 700 (1999).

[4] H. Linke, T. E. Humphrey, A. Löfgren, A. O. Sushkov, R. Newbury, R.P. Taylor, and P. Omling, Experimental Tunneling Ratchets, Science 286, 2314 (1999).

[5] J. E. Villegas, S. Savel'ev, F. Nori, E. M. Gonzalez, J. V. Anguita, R. García, and J.L. Vicent, A Superconducting Reversible Rectifier That Controls the Motion of Magnetic Flux Quanta, Science 302, 1188 (2003).

[6] M. Beck, E. Goldobin, M. Neuhaus, M. Siegel, R. Kleiner, and D. Koelle, High-Efficiency Deterministic Josephson Vortex Ratchet, Phys. Rev. Lett. 95, 090603 (2005).

[7] F. Falo, P. J. Martínez, J. J. Mazo, T. P. Orlando, K. Segall, and E. Trías, Fluxon Ratchet Potentials in Superconducting Circuits, Appl. Phys. A 75, 263 (2002).

[8] M. V. Costache and S. O. Valenzuela, Experimental Spin Ratchet, Science 330, 1645 (2010).

[9] P. Reimann, Brownian Motors: Noisy Transport Far from Equilibrium, Phys. Rep. 361, 57 (2002).
[10] P. Hänggi and F. Marchesoni, Artificial Brownian Motors: Controlling Transport on the Nanoscale, Rev. Mod. Phys. 81, 387 (2009).

[11] A. Ajdari, D. Mukamel, L. Peliti, and J. Prost, Rectified Motion Induced by ac Forces in Periodic Structures, J. Phys. I (France) 4, 1551 (1994).

[12] R. D. Astumian and P. Hänggi, Brownian Motors, Phys. Today 55, No. 11, 33 (2002).

[13] D. Poletti, T. J. Alexander, E. A. Ostrovskaya, B. Li, and Yu.S. Kivshar, Dynamics of Matter-Wave Solitons in a Ratchet Potential, Phys. Rev. Lett. 101, 150403 (2008).

[14] T. Salger, S. Kling, T. Hecking, C. Geckeler, L. MoralesMolina, and M. Weitz, Directed Transport of Atoms in a Hamiltonian Quantum Ratchet, Science 326, 1241 (2009).

[15] M. B. Tarlie and R. D. Astumian, Optimal Modulation of a Brownian Ratchet and Enhanced Sensitivity to a Weak External Force, Proc. Natl. Acad. Sci. U.S.A. 95, 2039 (1998).

[16] E. Zamora-Sillero, N. R. Quintero, and F. G. Mertens, Ratchet Effect in a Damped Sine-Gordon System with Additive and Parametric ac Driving Forces, Phys. Rev. E 74, 046607 (2006).

[17] R. Gommers, V. Lebedev, M. Brown, and F. Renzoni, Gating Ratchet for Cold Atoms, Phys. Rev. Lett. 100, 040603 (2008).

[18] W. Schneider and K. Seeger, Harmonic Mixing of Microwaves by Warm Electrons in Germanium, Appl. Phys. Lett. 8, 133 (1966).

[19] K. Seeger and W. Maurer, Nonlinear Electronic Transport in TTF-TCNQ Observed by Microwave Harmonic Mixing, Solid State Commun. 27, 603 (1978).

[20] M. Schiavoni, L. Sanchez-Palencia, F. Renzoni, and G. Grynberg, Phase Control of Directed Diffusion in a Symmetric Optical Lattice, Phys. Rev. Lett. 90, 094101 (2003).

[21] A. V. Ustinov, C. Coqui, A. Kemp, Y. Zolotaryuk, and M. Salerno, Ratchetlike Dynamics of Fluxons in Annular Josephson Junctions Driven by Biharmonic Microwave Fields, Phys. Rev. Lett. 93, 087001 (2004).

[22] R. Gommers, S. Bergamini, and F. Renzoni, DissipationInduced Symmetry Breaking in a Driven Optical Lattice, Phys. Rev. Lett. 95, 073003 (2005).

[23] S. Ooi, S. Savel'ev, M. B. Gaifullin, T. Mochiku, K. Hirata, and F. Nori, Nonlinear Nanodevices Using Magnetic Flux Quanta, Phys. Rev. Lett. 99, 207003 (2007).

[24] D. Cubero, V. Lebedev, and F. Renzoni, Current Reversals in a Rocking Ratchet: Dynamical versus SymmetryBreaking Mechanisms, Phys. Rev. E 82, 041116 (2010).

[25] F. Marchesoni, Harmonic Mixing Signal: Doubly Dithered Ring Laser Gyroscope, Phys. Lett. A 119, 221 (1986).

[26] I. Goychuk and P. Hänggi, Quantum Rectifiers from Harmonic Mixing, Europhys. Lett. 43, 503 (1998).

[27] S. Flach, O. Yevtushenko, and Y. Zolotaryuk, Directed Current due to Broken Time-Space Symmetry, Phys. Rev. Lett. 84, 2358 (2000).

[28] L. Morales-Molina, N. R. Quintero, F. G. Mertens, and A. Sánchez, Internal Mode Mechanism for Collective Energy Transport in Extended Systems, Phys. Rev. Lett. 91, 234102 (2003). 
[29] A. Engel, H.W. Müller, P. Reimann, and A. Jung, Ferrofluids as Thermal Ratchets, Phys. Rev. Lett. 91, 060602 (2003).

[30] S. Jäger and S.H.L. Klapp, Rotational Ratchets with Dipolar Interactions, Phys. Rev. E 86, 061402 (2012).

[31] R. Gommers, S. Denisov, and F. Renzoni, Quasiperiodically Driven Ratchets for Cold Atoms, Phys. Rev. Lett. 96, 240604 (2006).

[32] R. Gommers, M. Brown, and F. Renzoni, Symmetry and Transport in a Cold Atom Ratchet with Multifrequency Driving, Phys. Rev. A 75, 053406 (2007).

[33] X. Noblin, R. Kofman, and F. Celestini, Ratchetlike Motion of a Shaken Drop, Phys. Rev. Lett. 102, 194504 (2009).

[34] W. Wonneberger, Stochastic Theory of Harmonic Microwave Mixing in Periodic Potentials, Solid State Commun. 30, 511 (1979).

[35] M. Salerno and Y. Zolotaryuk, Soliton Ratchetlike Dynamics by ac Forces with Harmonic Mixing, Phys. Rev. E 65, 056603 (2002).

[36] H.-J. Breymayer, Harmonic Mixing in a Cosine Potential for Arbitrary Damping, Appl. Phys. A 33, 1 (1984).

[37] M. Borromeo, P. Hänggi, and F. Marchesoni, Transport by Bi-harmonic Drives: From Harmonic to Vibrational Mixing, J. Phys. Condens. Matter 17, S3709 (2005).

[38] N. R. Quintero, J. A. Cuesta, and R. Alvarez-Nodarse, Symmetries Shape the Current in Ratchets Induced by a Biharmonic Driving Force, Phys. Rev. E 81, 030102(R) (2010).

[39] A. V. Arzola, K. Volke-Sepúlveda, and J.L. Mateos, Experimental Control of Transport and Current Reversals in a Deterministic Optical Rocking Ratchet, Phys. Rev. Lett. 106, 168104 (2011).

[40] M. Schreier, P. Reimann, P. Hänggi, and E. Pollak, Giant Enhancement of Diffusion and Particle Selection in Rocked Periodic Potentials, Europhys. Lett. 44, 416 (1998).

[41] N. R. Quintero, R. Alvarez-Nodarse, and J. A. Cuesta, Ratchet Effect on a Relativistic Particle Driven by External Forces, J. Phys. A 44, 425205 (2011).
[42] A. Wouk, A Course of Applied Functional Analysis (Wiley, New York, 1979).

[43] In Eq. (2), it is implicitly assumed that if $n_{i}=0$ for some $i=1, \ldots, s$, variables $t_{i j}$ and factors $f\left(t_{i j}\right)$ are missing; e.g., for $s=2$ and $\mathbf{n}=(n, 0)$, the terms within the angular brackets are $c_{n, 0}\left(t_{11}, \ldots, t_{1 n}\right) f_{1}\left(t_{11}\right) \cdots f_{1}\left(t_{1 n}\right)$. With the same convention, $c_{\mathbf{0}}$ is just a constant.

[44] $\operatorname{gcd}\left(n_{1}, \ldots, n_{k}\right)$ stands for "greatest common divisor" of $n_{1}, \ldots, n_{k}$, i.e., the largest integer that divides all $n_{1}, \ldots, n_{k}$.

[45] The term Diophantine equation refers to an equation involving only integer numbers. It is named after Diophantus of Alexandria, who introduced them in his treatise Arithmetica.

[46] N. R. Quintero, B. Sánchez-Rey, and M. Salerno, Analytical Approach to Soliton Ratchets in Asymmetric Potentials, Phys. Rev. E 72, 016610 (2005).

[47] T. Wulf, C. Petri, B. Liebchen, and P. Schmelcher, Analysis of Interface Conversion Processes of Ballistic and Diffusive Motion in Driven Superlattices, Phys. Rev. E 86, 016201 (2012).

[48] S. Morito and H.M. Salkin, Using the Blankinship Algorithm to Find the General Solution of a Linear Diophantine Equation, Acta Informatica 13, 379 (1980).

[49] D. Cubero and F. Renzoni, Control of Transport in Two-Dimensional Systems via Dynamical Decoupling of Degrees of Freedom with Quasiperiodic Driving Fields, Phys. Rev. E 86, 056201 (2012).

[50] R. Gommers, P. Douglas, S. Bergamini, M. Goonasekera, P. H. Jones, and F. Renzoni, Resonant Activation in a Nonadiabatically Driven Optical Lattice, Phys. Rev. Lett. 94, 143001 (2005).

[51] L. Morales-Molina, N. R. Quintero, A. Sánchez, and F. G. Mertens, Soliton Ratchets in Homogeneous Nonlinear Klein-Gordon Systems, Chaos 16, 013117 (2006).

[52] Y. Zolotaryuk and M. Salerno, Discrete Soliton Ratchets Driven by Biharmonic Fields, Phys. Rev. E 73, 066621 (2006).

[53] R. Bartussek, P. Hänggi, and J. G. Kissner, Periodically Rocked Thermal Ratchets, Europhys. Lett. 28, 459 (1994). 